\title{
Effect of Nitric Oxide Bioavailability on Cutaneous Microvascular Function in Healthy Young African Americans
}

\author{
Kim K, Hurr C, Brothers RM
}

Environmental and Autonomic Physiology Laboratory; Department of Kinesiology and Health Education; University of Texas at Austin; Austin, TX

Category: Doctoral

Advisor / Mentor: R. Matthew Brothers (r.m.brothers@austin.utexas.edu)

We have recently demonstrated impaired microcirculatory function in healthy young African Americans (AAs) as indexed by attenuated cutaneous vasodilation during local heating. The mechanisms resulting in this impairment are multifactorial; however, recent evidence suggests that decreased nitric oxide (NO) bioavailability is a primary contributor. PURPOSE: To expand our previous findings and determine if this reduced microvascular vasodilatory response in AAs is related to a decrease in NO bioavailability. We hypothesized that NO dependent cutaneous vasodilation in response to local heating is lower in healthy young AAs relative to age / sex / body mass matched Caucasians (CA). METHOD: To date 9 healthy CAs $(24 \pm 3 \mathrm{yrs})$ and 9 AAs $(23 \pm 3 \mathrm{yrs})$ have participated in this study. Two intradermal microdialysis membranes were inserted in the non-dominant forearm. At each site skin blood flow was indexed via a laser Doppler flow probe housed within a local heater. After recovery from the insertion trauma, the local heaters were clamped at $33^{\circ} \mathrm{C}$ and each microdialysis site was randomly assigned to receive either Lactated Ringer's solution as a control or $20 \mathrm{mM}$ NG-nitro-L-arginine (L-NAME) to inhibit NO. After 45 min, skin temperature was increased to $42{ }^{\circ} \mathrm{C}$ for $30 \mathrm{~min}$ while each site continued to receive their solutions. Finally, site specific maximal cutaneous vasodilation was achieved by a combination of infusion of $28 \mathrm{mM}$ sodium nitroprusside and $43^{\circ} \mathrm{C}$ heating. Cutaneous vascular conductance (CVC) was calculated as skin blood flow/mean arterial pressure and normalized as \% maximal CVC (\%CVCmax). The \%CVCmax at L-NAME site was subtracted from the \%CVCmax at the control site to assess the NO contribution. A two-way mixed-model ANOVA was used for data analysis. RESULTS: NO contribution was not different during $33{ }^{\circ} \mathrm{C}(4 \pm 4$ vs. $4 \pm 4 \%$ CVCmax, $\mathrm{p}=0.92)$; however, the NO contribution to vasodilation was reduced during $42{ }^{\circ} \mathrm{C}$ heating in the AA (CA: $57 \pm 14$ vs. AA: $44 \pm 7 \%$ CVCmax, $p=0.03$ ). As a result the NO contribution during local heating was attenuated in the AA (Interaction: Temp*Race, $\mathrm{p}$ $=0.02$ ). CONCLUSION: NO dependent cutaneous vasodilation in response to local heating was significantly different between AAs and CAs. Therefore, this finding suggests that decreased NO bioavailability may cause impaired cutaneous microvascular function in AAs.

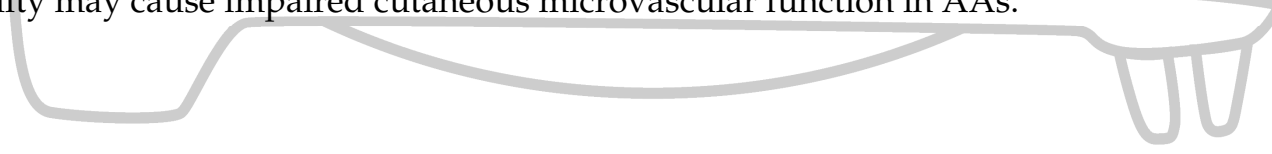

